\title{
A Simplified Scheme of Estimation and Cancellation of Companding Noise for Companded Multicarrier Transmission Systems
}

\author{
Siming PENG ${ }^{1}$, Zhigang YUAN ${ }^{1}$, Jun YOU ${ }^{2}$, Yuehong SHEN ${ }^{1}$, Wei JIAN ${ }^{1}$ \\ ${ }^{1}$ Dept. of Wireless Communications, PLA University of Science and Technology, 210014 Nanjing, China \\ ${ }^{2}$ Dept. of Command Information System, PLA University of Science and Technology, 210014 Nanjing, China \\ lgdxpsm@gmail.com, yzhigang_cn@163.com, chunfeng22259@126.com
}

\begin{abstract}
Nonlinear companding transform is an efficient method to reduce the high peak-to-average power ratio (PAPR) of multicarrier transmission systems. However, the introduced companding noise greatly degrades the bit-errorrate (BER) performance of the companded multicarrier systems. In this paper, a simplified but effective scheme of estimation and cancellation of companding noise for the companded multicarrier transmission system is proposed. By expressing the companded signals as the summation of original signals added with a companding noise component, and subtracting this estimated companding noise from the received signals, the BER performance of the overall system can be significantly improved. Simulation results well confirm the great advantages of the proposed scheme over other conventional decompanding or no decompanding schemes under various situations.
\end{abstract}

\section{Keywords}

Multicarrier transmission systems, peak-to-average power ratio (PAPR), nonlinear companding transform (NCT), companding noise cancellation

\section{Introduction}

Multicarrier transmission is a promising technique in future communication systems. However, one of the major drawbacks of multicarrier systems is the inherent high peak-to-average power ratio (PAPR) of the transmitted signals. It's known that the efficiency of the high power amplifier (HPA) is directly related to the PAPR of the input multicarrier signals especially in the orthogonal frequency division multiplexing (OFDM) systems, which is applied in many important wireless communication standards such as the Third Generation Partnership Project (3GPP) Long-Term Evolution Advanced (LTE-A) standard [1], [2]. The PAPR problem still prevents OFDM from being adopted in the uplink of wireless communication standards [1].

Up to now, many works have been conducted to deal with this high PAPR problem [3]. Such as the iterative clipping and filtering [4], [5], [6], coding [7], Partial Transmission Sequence (PTS) [8], [9], [10], Selective Mapping (SLM) [11], Tone Reservation (TR) [12], companding transform (CT) [13]-[20] and so on. Among them, the clipping and companding may be the simplest two methods, since they can be employed directly to the multicarrier systems without any restrictions on the number of subcarriers and frame format and so on. However, due to the clipping often introduces serious in-band distortions as well as out-ofband radiation, consequently, the bit-error-rate (BER) performance of the system is greatly degraded. On the contrary, the companding transform can not only achieve more effective PAPR reduction but also better BER performance than the clipping method. Hence, it attracts more and more researchers' attention in recent years.

Since companding transform is an extra predistortion process employed on the original signals, hence, the introduced companding noise may also degrade the system performance to some extend. Conventional decompanding operation can approximately remove the companding noise at the receiver [13], [14], [15], [16], but the channel noise will be amplified by the decompanding function simultaneously, and consequently, the system performance will not be so optimistic especially under low signal-to-noise ratio (SNR) region. In order to avoid amplifying the channel noise caused by the decompanding operations, in [17], the authors proposed to abandon the decompanding operation at the receiver, and although a great BER performance improvement can be achieved, however, there is still a relative large gap of BER performance away from the performance bound. In [19], the authors proposed an iterative receiver to estimate and cancel the companding noise. By referring to the Bussgang theorem, the companding signals are regarded as the summation of a useful attenuated input replica and an uncorrected nonlinear distortion noise, and then removed from the receiver. Although the BER performance can be greatly improved in this scheme, however, due to the inherent complex expression of the companding function, the accurate attenuation coefficient of the input signals will difficultly to be determined, and consequently, greatly restrains the effectiveness for general companding transforms. 
In this paper, a simplified scheme of estimation and cancellation of nonlinear companding noise is proposed. By expressing the companded signals as the summation of the companding noise added to the original signals, and then, subtracting the estimated companding noise from the receiver, the above difficulties of analysis and calculation of the attenuation coefficient of the original signals can be well resolved. Moreover, a significant BER performance improvement than conventional operations, such as the decompanding or no decompanding, at the receiver can be achieved simultaneously. It also shows that the presented scheme is robust in various practical situations.

The rest of this paper is organized as follows. In Sec. 2, a typical multicarrier system model is described and the PAPR problem is formulated briefly. The theoretical analysis of the proposed scheme is presented in Sec. 3. In the next section, the overall BER performance of a typical exponential companding (EC) equipped with the proposed companding noise cancellation scheme is evaluated and followed by the conclusion summarized in Sec. 5 .

\section{System Model}

Fig. 1 shows the block diagram of a typical multicarrier transmission system with companding transform. Let us denote the data symbols $X_{k}, k=0,1, \ldots, N-1$, as a vector $\mathbf{X}=\left[X_{0}, X_{1}, \ldots, X_{N-1}\right]^{T}$ with $N$ subcarriers, where $(\cdot)^{T}$ is the matrix transpose operation. The complex baseband representation of a multicarrier signal is given by

$$
x(t)=\frac{1}{\sqrt{N}} \sum_{k=0}^{N-1} X_{k} e^{j 2 \pi k \Delta f t}, 0 \leqslant t \leqslant N T_{s}
$$

where $j=\sqrt{-1}, \Delta f$ is the subcarrier interval, and $N T_{s}$ is the useful data block period. In general, the subcarriers are chosen to be orthogonal (i.e. $\Delta f=1 / N T_{S}$ ).

Generally, the PAPR of multicarrier signals $x(t)$ is defined as the ratio between the maximum instantaneous power and its average power, i.e.

$$
P A P R=\frac{\max _{0 \leqslant t \leqslant N T}\left[|x(t)|^{2}\right]}{1 / N T \int_{0}^{N T}|x(t)|^{2} d t} .
$$

To better approximate the PAPR of continuous-time OFDM signals, the OFDM signals samples are obtained by $L$ times oversampling. $L$-times oversampled time-domain samples can be achieved by performing a $L N$-point IFFT of the data block with $(L-1) N$ zero-padding, i.e.

$$
\mathbf{X}_{p}=[X_{0}, \ldots, X_{\frac{N}{2}-1}, \underbrace{0, \ldots, 0}_{(L-1) N}, X_{\frac{N}{2}}, \ldots, X_{N-1}]^{T} .
$$

Therefore, the oversampled IFFT output can be expressed as

$$
x(n)=\frac{1}{\sqrt{N}} \sum_{k=0}^{N-1} X_{k} e^{j \frac{2 \pi n k}{L N}}, 0 \leq n \leq L N-1 .
$$

The corresponding PAPR computed from the $L$-times oversampled time domain OFDM signal sample is defined as

$$
P A P R=\frac{\max _{0 \leq n \leq L N-1}\left[|x(n)|^{2}\right]}{\mathbb{E}\left[|x(n)|^{2}\right]}
$$

where $\mathbb{E}[\cdot]$ denotes the expectation operator.

Assume that the input information symbols are statistically independent and identically distributed. Based on the central limit theory, $x(n)$ can be approximated as a complex Gaussian process with zeros mean and variance $\sigma^{2}$ when the number of sub-carriers $N$ is large enough (e.g. $N \geqslant 64$ ). Thus, the amplitude $|x(n)|$ follows a Rayleigh distribution with the probability distribution function (PDF) as

$$
f_{\left|x_{n}\right|}=\frac{2 x}{\sigma^{2}} \exp \left(-\frac{x^{2}}{\sigma^{2}}\right), x \geqslant 0 .
$$

From (6), we can see that the peak power of $\left|x_{n}\right|$ can take a value much large than its average power. That is to say, the multicarrier signal has a large PAPR, which leads to a negative impact on the system performance. While, by reallocating the power or the statistics of multicarrier signal reasonable, the companding transform can well resolve this high PAPR problem.

The fundamental principle of nonlinear companding transform can be described as follows [14]. The original signal $x(n)$ is companded before converted into analog waveform and amplified by the HPA. The companded signal is denoted as

$$
y_{n}=\zeta\left(x_{n}\right)
$$

where $\zeta(\cdot)$ is the companding function which only changes the amplitude of $x_{n}$. When passing through the AWGN channel, the transmitted signals can be recovered by the corresponding decompanding function $\zeta^{-1}(\cdot)$, i.e.

$$
\tilde{x}_{n}=\zeta^{-1}\left(y_{n}+w_{n}\right) \approx x_{n}+\zeta^{-1}\left(w_{n}\right)
$$

where $w_{n}$ is the channel noise.

It has been pointed in [17] that the BER performance of companded multicarrier systems can be improved by carefully design the companding function $\zeta(\cdot)$ [13]-[20], however, due to the amplified channel noise (by decompanding operation) or unprocessed companding noise (by no decompanding operation), this modification may be limited in practice. Hence, there is considered to be a trade-off between PAPR reduction and BER performance for companding transform. However, in the subsequent works, we will show that this problem can be well resolved by the proposed companding noise cancellation scheme. 


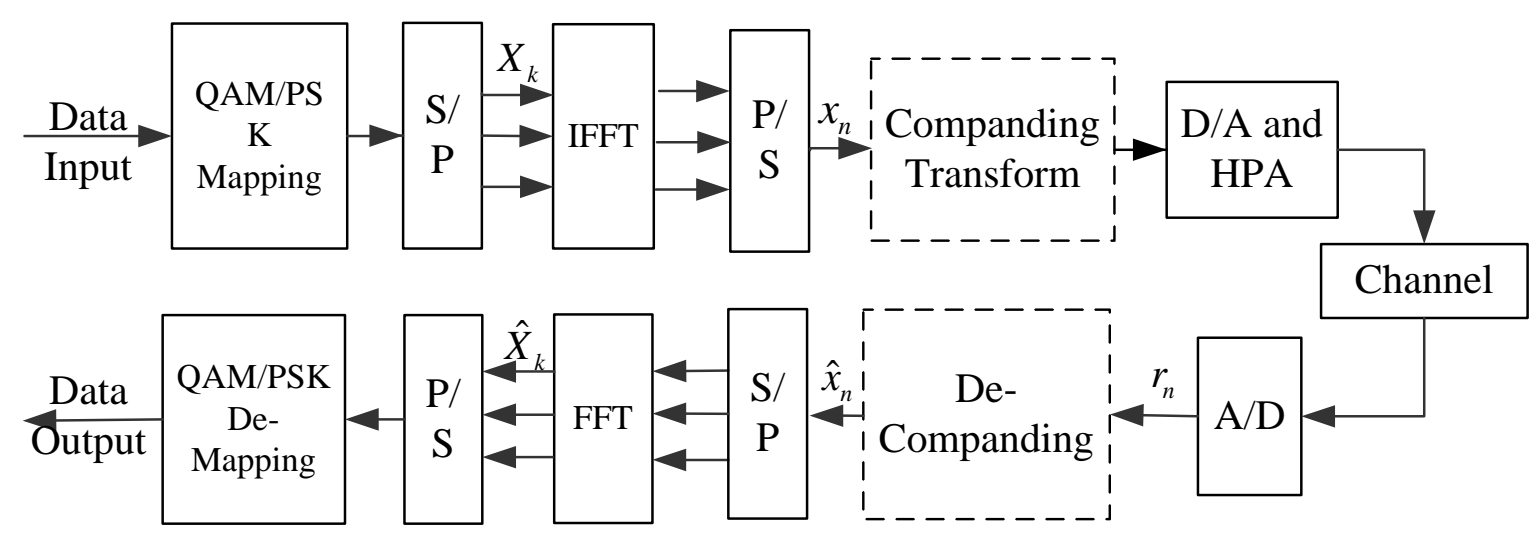

Fig. 1. Block diagram of typical multicarrier transmission system with companding transform.

\section{Algorithm Formulation}

In this section, we will review the basic concepts of conventional Bussgang based companding noise cancelllation scheme briefly, and then, the proposed scheme will be presented subsequently.

\subsection{Bussgang Theorem Based Scheme}

According to the Bussgang theorem [19], the companded multicarrier signal $y(n)$ can be modeled as the aggregate of an attenuated signal component and companding noise $d_{n}$, i.e.

$$
y(n)=\alpha x(n)+d_{n}, n=0,1, \ldots, L N-1
$$

where $\alpha$ is the attenuate coefficient, and which is a time invariant for stationary input processes.

From (9), the attenuate coefficient $\alpha$ can be calculated as

$$
\alpha=\frac{\mathbb{E}\left\{y(n) x^{*}(n)\right\}}{\mathbb{E}\left\{x(n) x^{*}(n)\right\}}=\frac{1}{\sigma^{2}} \int_{0}^{\infty} x \cdot \zeta(x) \cdot f_{|x|}(x) d x
$$

where $f_{|x|}(x)$ is as defined in (6) and $x^{*}(t)$ is the complex conjugate of $x(t)$.

In the receiver, by making full use of the received signal $y(n)$ and reconstructing the companding process as that of the transmitter, the estimated companding noise component can be calculated as

$$
\hat{d}_{n}=\hat{y}(n)-\alpha \hat{x}(n), n=0,1, \ldots, L N-1
$$

where $\hat{y}(n), \hat{x}(n)$ are the reconstructed companded signal and the detected signal at the receiver, respectively.

However, it should be noted that the companding function $\zeta(\cdot)$ in (10) often has a complex expression (for example, see the companding functions in [14], [15] and [17]), which make the coefficient $\alpha$ difficult to be accurately calculated for general companding transforms. Moreover, the precision of $\alpha$ will have a great impact on the ultimate system performance. Hence, the effectiveness of conventional estimation and cancellation of companding noise will be greatly restrained in practice.

\subsection{Proposed Scheme}

Different from (9), if the attenuation of original signals is regarded as caused by the companding noise, then the output of the compander can be simply expressed as the original signals added with an extra companding noise component, i.e.

$$
y(n)=x(n)+\hat{d}_{n}, n=0,1, \ldots, L N-1
$$

where $\hat{d}_{n}$ is the equivalent companding noise.

Hence, the received signal can be expressed as

$$
\begin{aligned}
r(n) & =h(n) * y(n)+w_{n} \\
& =h(n) * x(n)+h(n) * \hat{d}_{n}+w_{n}, n=0,1, \ldots, L N-1
\end{aligned}
$$

where ' $*$ ' is the convolution operation and $h(n)$ is the impulse response of the transmitting channel.

Assume the detected symbol is $\tilde{X}_{k}$ (or equivalently $\tilde{x}(n))$, and the output of the compander at the receiver is $\tilde{y}(n)$, i.e.

$$
\tilde{y}(n)=\tilde{x}(n)+\tilde{d}_{n}, n=0,1, \ldots, L N-1
$$

where $\tilde{d}_{n}$ is the companding noise regenerated at the receiver.

Since $\tilde{y}(n), \tilde{x}(n)$ are all achievable at the receiver, hence, the companding noise can be estimated as

$$
\tilde{d}_{n}=\tilde{y}(n)-\tilde{x}(n), n=0,1, \ldots, L N-1 .
$$

According to current channel estimation $\tilde{h}(n)$, then, $\tilde{h}(n) * \tilde{d}_{n}$ is subtracted from the current channel observation $r(n)$ to obtain the refined channel observation $\hat{r}(n)$, i.e. 


$$
\begin{aligned}
\hat{r}(n) & =r(n)-\tilde{h}(n) * \tilde{d}_{n} \quad n=0,1, \ldots, L N-1 \\
& =h(n) * x(n)+\left(h(n) * \hat{d}_{n}-\tilde{h}(n) * \tilde{d}_{n}\right)+w_{n} .
\end{aligned}
$$

It can be seen from (16) that the channel estimation error between $\tilde{h}(n)$ and $h(n)$ may affect the ultimate result of $\hat{r}(n)$, however, this error may also affect the estimation precision of $\tilde{d}_{n}$ (due to the decision error of $\tilde{X}_{k}$ ). On the other hand, when $\tilde{h}(n)$ severe deviates from $h(n)$, then, the channel estimation error will become the dominate interference component. Hence, we will mainly consider the approximate ideal channel estimation, i.e. $\tilde{h}(n) \approx h(n)$ in the subsequent works.

When $\tilde{d}_{n} \rightarrow \hat{d}_{n}$, then we will have the asymptotic ideal ${ }^{1}$ result, i.e.

$$
\hat{r}_{n} \rightarrow h(n) * x(n)+w_{n}, n=0,1, \ldots, L N-1 .
$$

The above processes can be summarized in Fig. 2 . that

Comparing (9) and (12), we can have the conclusions

- The proposed scheme is more simple than that of the conventional estimation and cancellation of companding noise scheme which bases on the Bussgang theorem [19]. Moreover, since the calculation of the attenuated coefficient $\alpha$ can be avoided in the presented scheme, hence, it can be easily adopted to general companding schemes.

- The novel scheme is more robust than that of the conventional scheme. This is due to the estimated companding noise in the presented scheme will not be affected by the precision of $\alpha$ as that in the conventional scheme.

\section{Simulation Results}

To evaluate the overall system performance, computer simulations are performed based on an OFDM system with $N=256$ subcarriers and the input bit stream is modulated by Quaternary Phase Shift Keying (QPSK) and 16 Quadrature Amplitude Modulation (16QAM). The AWGN channel as well as frequency-selective multi-path fading channel [15] is considered in the simulations. Moreover, the oversampling factor $L=4$ and a cycle prefix with the length of $1 / 4$ symbol is employed to mitigate the inter-symbol interference. Furthermore, for the convenience of comparison, a typical nonlinear companding function, i.e. the EC scheme proposed in [13] with companding function ${ }^{2}$

$$
\zeta(x)=\operatorname{sgn}(x) \sqrt[d]{v\left[1-\exp \left(-\frac{|x|^{2}}{\sigma^{2}}\right)\right]}
$$

where

$$
v=\left(\frac{\mathbb{E}\left[|x|^{2}\right]}{\mathbb{E}\left[\sqrt[d]{\left[1-\exp \left(-\frac{|x|^{2}}{\sigma^{2}}\right)\right]^{2}}\right]}\right)^{\frac{d}{2}}
$$

and $d$ is the companding degree (the PAPR performance of EC according to [13] with different companding degree $d$ is summarized in Tab. 1), is equipped with several conventional operations at the receiver, including conventional decompanding (DC) [13], [14], [15], [16], [20], no decompanding (NDC) [17], [18] as well as the proposed companding noise cancellation $(\mathrm{CNC})$ operations are all considered in the simulations.

\begin{tabular}{|c|c|c|c|}
\hline & \multicolumn{2}{|c|}{ EC } & Original signals \\
\hline PAPR $(\mathrm{dB})$, & $d=1$ & $d=2$ & \\
$\mathrm{CCDF}=10^{-3}$ & 4.98 & 3.21 & 11.38 \\
\hline
\end{tabular}

Tab. 1. PAPR performance of EC with different companding degrees.

\subsection{BER in AWGN Channel}

Figure 3 shows the BER performance of OFDM system with QPSK modulation employing EC and equipped with different operations at the receiver over AWGN channel. In this figure, the curve of "Performance bound" is the signals transmitted without living through any nonlinear distortions. Given that the BER at $P_{e}=10^{-5}$, we can find that the companded signals are suffering from a serious nonlinear distortions especially when with conventional decompanding operations at the receiver, and there is a $3.23 \mathrm{~dB} \varepsilon_{b} / N_{0}$ gap between EC-DC with $d=1$ and the performance bound and more than $6 \mathrm{~dB} \varepsilon_{b} / N_{0}$ gap for EC-DC with $d=2$. While, although there is a significant BER improvement when with no decompanding operation at the receiver, however, there is still a gap of $0.78 \mathrm{~dB} \varepsilon_{b} / N_{0}$ at EC-NDC with $d=1$ and $3.03 \mathrm{~dB} \varepsilon_{b} / N_{0}$ at EC-NDC with $d=2$ away from the performance bound. However, when with the proposed companding noise cancellation scheme, the BER gap between EC-CNC and the performance bound can be effectively restored to no more than $0.29 \mathrm{~dB}$ regardless of the companding degree $d$.

Figure 4 shows the BER performance of EC with 16QAM modulation and different operations at the receiver over AWGN channel. It's obvious that the overall BER performance has suffering a more serious degradation than that of with QPSK modulation. Furthermore, when with EC-DC and $d=1$, there is a $5.58 \mathrm{~dB} \varepsilon_{b} / N_{0}$ gap away from the performance bound. While, we can also find that when with high $\varepsilon_{b} / N_{0}$, the conventional decompanding operation will trend to outperform that with no decompanding operation at

\footnotetext{
${ }^{1}$ As we will show later, this is due to the inherent estimated errors of the detected signals $\tilde{x}(n)$, and this error is unavoidable but can be improved by some other techniques, such as the channel coding.

${ }^{2}$ It's obvious that (10) is not appropriate for this companding function, since the coefficient $v$ in (18) can only be calculated numerically, and this phenomenon is widely exits in some other companding functions (see [14]-[20]).
} 


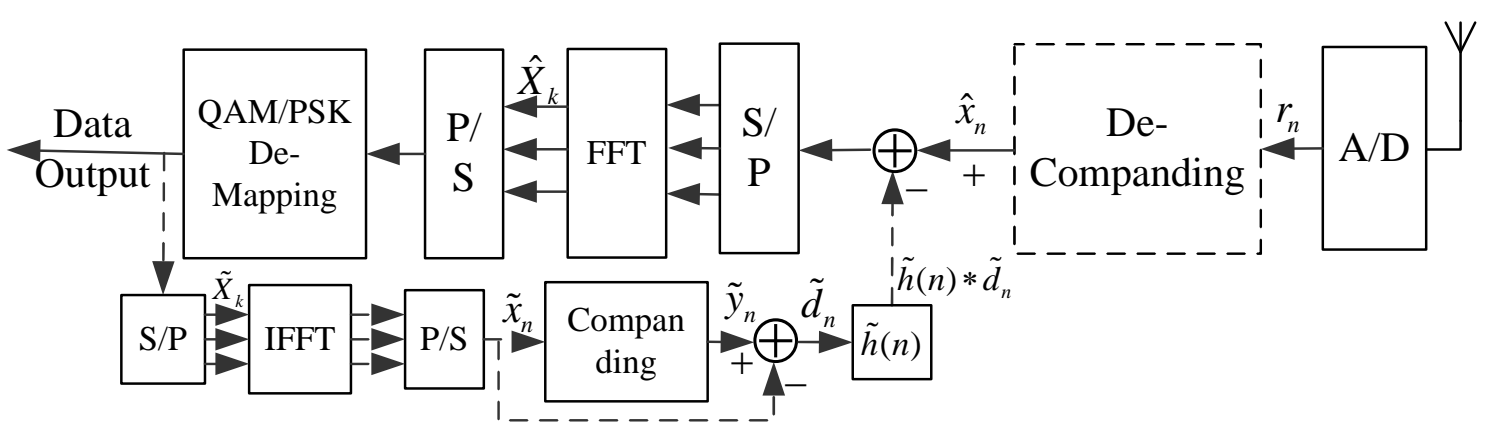

Fig. 2. Block diagram of the proposed scheme for companding transform. Note that when the SNR is low, it's prefer to with no decompanding at the receiver, and then $\hat{x}(n)=r(n)$.

the receiver. This is due to that although the decompanding operation may amplifier the channel noise, while, when $\varepsilon_{b} / N_{0}$ is high enough (e.g. $\varepsilon_{b} / N_{0}>18 \mathrm{~dB}$ ), the companding noise will become the dominant interference component and must be removed from the received signals. When with the proposed companding noise cancellation scheme, there is a $1.09 \mathrm{~dB} \varepsilon_{b} / N_{0}$ gap between EC-CNC with $d=2$ and the performance bound, while the relative gap is only $0.16 \mathrm{~dB}$ for EC-CNC with $d=1$, which further demonstrates the great advantages of the proposed scheme over other conventional operations.

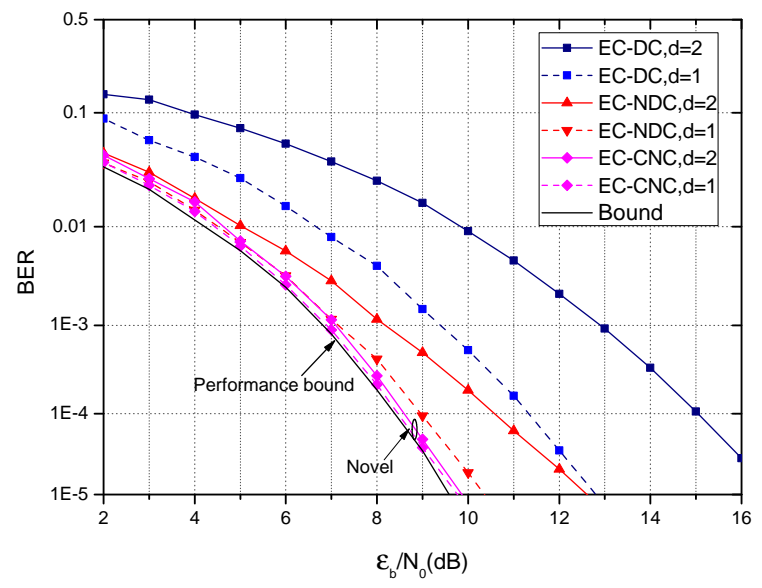

Fig. 3. BER performance of QPSK modulation and EC equipped with different operations over AWGN channel.

\subsection{BER with HPA over AWGN Channel}

For most wireless communication systems, the HPA is widely used to provide adequate transmit power. In this paper, the solid state power amplifier (SSPA) model described in [21] is considered in the subsequent works. Fig. 5 shows the BER performance of different operations at the receiver with SSPA and $I B O=0 \mathrm{~dB}$ over AWGN channel. It is seen that after passing through the SSPA, the BER performance has been degraded to some extent. In this figure, the "Original signals" is the signals without companding transform and directly transmitted to the SSPA, consequently, result in $3.33 \mathrm{~dB} \varepsilon_{b} / N_{0}$ degradation compared to the performance bound as shown in Fig. 3. Moreover, we can also find that the EC-CNC with $d=2$ outperforms the original signals 2.55 $\mathrm{dB}$ and $0.16 \mathrm{~dB}$ better than EC-CNC with $d=1$.

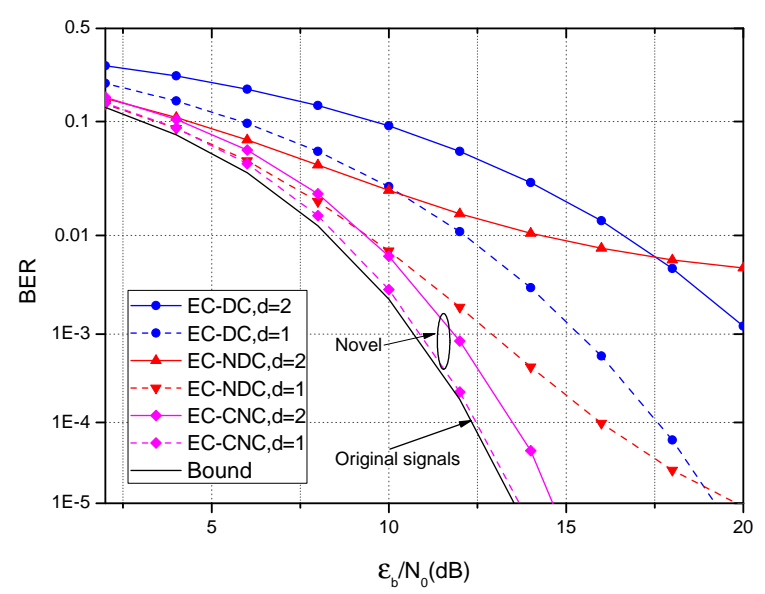

Fig. 4. BER performance 16QAM modulation of EC equipped with different operations at the receiver over AWGN channel.

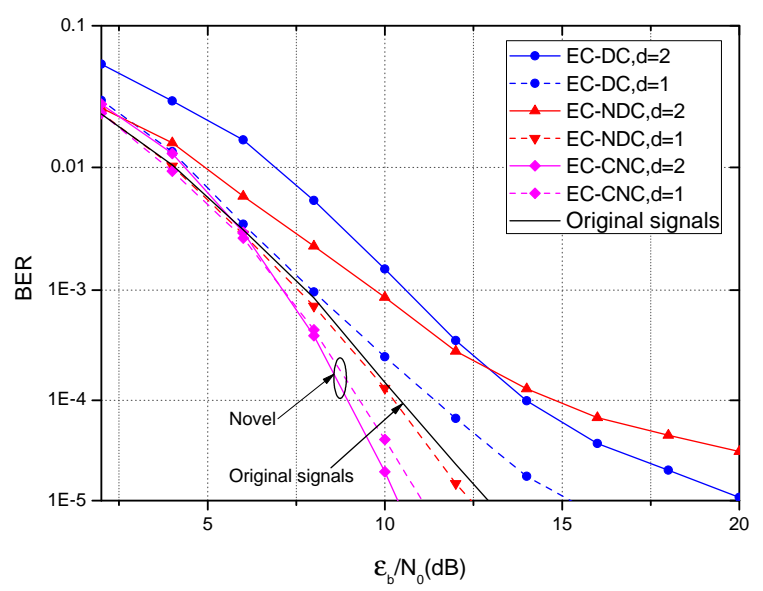

Fig. 5. BER performance of EC with HPA over AWGN channel with different operations at the receiver. 


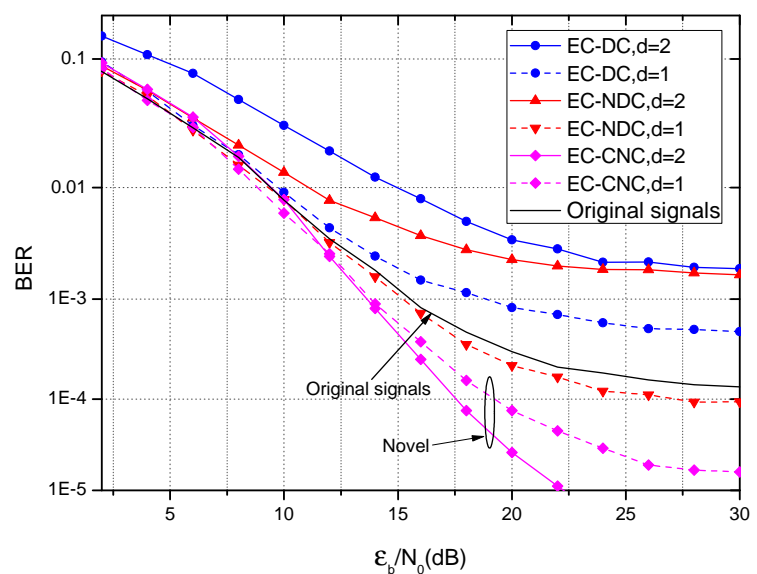

Fig. 6. BER performance of the receiver with HPA and equipped with various operations over fading channel.

\subsection{BER with HPA over Fading Channel}

Figure 6 shows the BER performance of EC with SSPA and equipped with different operations at the receiver over fading channel. In this figure, the IEEE 802.16 fading channel model described in [15] is considered in the simulations. From it we can see that different with other conventional operations at the receiver, the EC equipped with $\mathrm{CNC}$ greatly outperforms the original signals. Furthermore, comparing Fig. 6 and Fig. 5, we can also find that the advantages of the proposed scheme are more significant in practical wireless communication systems than that of in the AWGN channel.

\subsection{BER with Iterative Filtering}

Since the companding operation is a type of nonlinear process that may lead to out-of-band radiation, hence, an iterative companding and filtering technique may need to remove the out-of-band radiation and peak regrowth. However, it's known that the filtering may lead to the degradation of the BER performance, hence, it's necessary to estimate the practical BER performance of companding transform when considering the iterative filtering technique.

In this subsection, we mainly employ filtering in the baseband signals in the frequency domain [4], and the filter is based on the rectangular window, and can be defined as

$$
\mathbf{Y}_{f}=\mathbf{H}_{f} \mathbf{Y}_{c}
$$

where $\mathbf{Y}_{c}$ is the companded signal in frequency domain, $\mathbf{Y}_{f}$ is the filtered signals, and $\mathbf{H}_{f}$ is the frequency response of the filter defined by

$$
H_{f}(k)=\left\{\begin{array}{l}
1,0 \leqslant k \leqslant N / 2-1, L-N / 2 \leqslant k \leqslant L-1 \\
0, \text { otherwise }
\end{array}\right.
$$

Figure 7 shows the BER performance of EC over AWGN channel with iterative filtering at the transmitter and equipped different operations at the receiver with 2 iterations. Note that the receiver also performs the same iterative companding and filtering process as that at the transmitter. From it we can see that although the SNR gap between $\mathrm{EC}-\mathrm{CNC}$ and the performance bound is slightly increased to $0.2 \mathrm{~dB}$ at $d=1$ and $0.98 \mathrm{~dB}$ at $d=2$ compared with Fig. 3, however, it's obvious that the proposed scheme still greatly outperforms other conventional schemes, which well verifies the robustness of the proposed scheme under various situations.

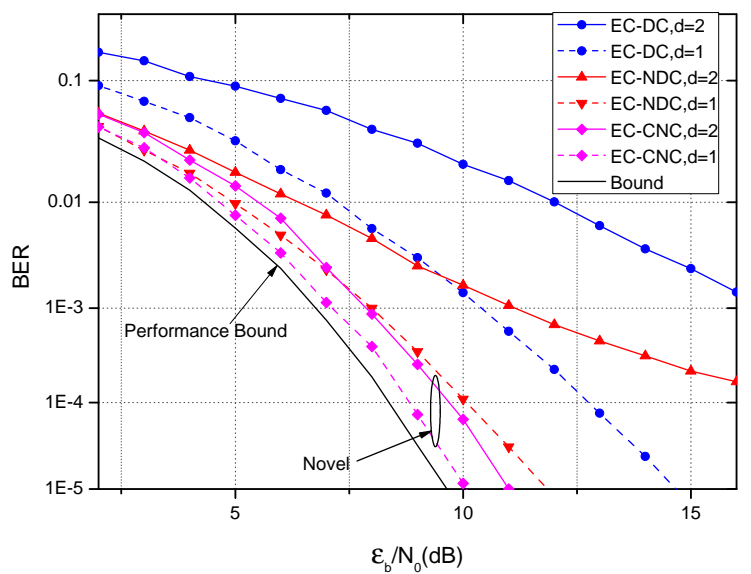

Fig. 7. BER performance of EC with iterative filtering and equipped with various operations at the receiver over AWGN channel.

\section{Conclusion}

Nonlinear companding noise in companded multicarrier systems greatly degrades the BER performance of the overall system. In this paper, a simplified but effective scheme of estimation and cancellation of companding noise is proposed to enhance the BER performance of the companded multicarrier systems. By expressing the companding process as the original signals added with a companding noise component and removing the estimated companding noise from the receiver, a great BER performance improvement can be achieved compared to conventional operations. Although with a slightly increased complexity (due to additional IFFT/FFT and companding operations at the receiver), we believe that the proposed scheme may well resolve the embarrassment, i.e. the trade-off problem between PAPR reduction and BER performance, for the conventional companding schemes.

\section{Acknowledgements}

This work was supported by the National Natural Science Foundation of China (grant nos 60772083 and 61201242); The Natural Science Foundation of Jiangsu Province (grant no. BK2012057) and the PLA University Preresearch Foundation (KYTYZLXY1208). 


\section{References}

[1] WUNDER, G., FISHCHER, R. F. H., BOCHE, H., ET AL. The PAPR problem in OFDM transmission: new direction for longlasting problem. IEEE Signal Processing Magazine, 2013, vol. 51, no. 11, p. 130-144. DOI: 10.1109/MSP.2012.2218138

[2] POLAK, L., KRATOCHVIL, T. Exploring of the DVB-T/T2 performance in advanced mobile TV fading channels. In Proc. 36th International Conference on Telecommunictaions and Signal Processing (TSP2013), Rome (Italy), 2013, p. 768-772. DOI: 10.1109/TSP.2013.6614042

[3] HAN, S. H., LEE, J. H. An overview: peak-to-average power ratio reduction techniques for multicarrier transmission. IEEE Wireless Communications, 2004, vol. 12, no. 2, p. 56-65. DOI: 10.1109/MWC.2005.1421929

[4] ZHU, X., PAN, W., LI, H., ET AL. Simplified approach to optimized iterative clipping and filtering for PAPR reduction of OFDM signals. IEEE Transactions on Communications, 2013, vol. 61, no. 5, p. 1891-1901. DOI: 10.1109/TCOMM.2013.021913.110867

[5] CHEN, H., HAIMOVICH, A., M. Iterative estimation and cancellation of clipping noise for OFDM signals. IEEE Coтmunications Letters, 2003, vol. 7, no. 7, p. 305-307. DOI: 10.1109/LCOMM.2003.814720

[6] XIA, L., LI, Z., YOUXI, T., ET AL. Analysis of the performance of iterative estimation and cancellation of clipping non-linear distortion in OFDM. In Proceeding of Future Generation of Communications and Networking, 2007, p. 1-5. DOI: 10.1109/FGCN.2007.68

[7] SLMANE, S., B. Reduction the peak-to-average power ratio of OFDM signals through precoding. IEEE Transactions on Vehicular Technology, 2007, vol. 56, no. 2, p. 686-695. DOI: 10.1109/TVT.2007.891409

[8] QI, X., LI, Y, HUANG., H. A low complexity PTS scheme based on tree for PAPR reduction. IEEE Communications Letters, 2012, vol. 16, no. 9, p. 1486-1488. DOI: 10.1109/LCOMM.2012.072012.121228

[9] VARAHRAM, P., ALI, M., B. A low complexity partial transmit sequence for peak to average power ratio reduction in OFDM systems. Radioengineering, 2011, vol. 20, no. 3, p.677-682.

[10] MARSALEK, R. On the reduced complexity interleaving method for OFDM PAPR reduction. Radioengineering, 2006, vol. 15, no. 3, p. 49-53.

[11] JIANG, T., NI, C., GUAN, L. A novel phase offset SLM scheme for PAPR reduction in Alamouti MIMO-OFDM systems without side information. IEEE Signal Processing Letters, 2013, vol 20, No. 4, p. 383-386. DOI: 10.1109/LSP.2013.2245119

[12] WANG, L., TELLAMBURE, C. Analysis of clipping noise and tone reservation algorithms for peak reduction in OFDM systems. IEEE Transactions on Vehicular Technology, 2008, vol. 57, no. 3, p. 16751694. DOI: $10.1109 /$ TVT.2007.907282

[13] JIANG, T., YANG, Y., SONG, Y., H. Exponential companding technique for PAPR reduction in OFDM systems. IEEE Transactions on Broadcasting, 2005, vol. 51, no. 2, p. 244-248. DOI: 10.1109/TBC.2005.847626

[14] WANG, Y., GE, J., WANG, L., ET AL. Nonlinear companding transform for reduction of peak-to-average power ratio in OFDM systems. IEEE Transactions on Broadcasting, 2013, vol. 59, no. 2, p. 369375. DOI: $10.1109 /$ TBC.2012.2219252

[15] JENG, S., S., CHEN, J., M. Effective PAPR reduction in OFDM systems based on a companding technique with trapezium distribution. IEEE Transactions on Broadcasting, 2010, vol. 56, no. 2, p. 258262. DOI: 10.1109/TBC.2011.2112237
[16] PENG, S., SHEN, S., YUAN, Z., ET AL. A novel nonlinear companding transform for PAPR reduction in lattice-OFDM systems. Frequenz, 2014, vol. 69, no. 2, p. 461-469. DOI: 10.1515/freq-20130169

[17] HOU, J., GE, J., ZHAI, D.,ET AL. Peak-to-average power ratio reduction of OFDM signals with nonlinear companding scheme. IEEE Transactions on Broadcasting, 2010, vol. 56, no. 2, p. 258-262. DOI: 10.1109/TBC.2010.2046970

[18] PENG, S., SHEN, Y., YUAN, Z., ET AL. PAPR reduction of LOFDM signals with an efficient nonlinear companding transform. In Proceeding of International Conference on Wireless Communications and Signal Processing, WCSP'13, Hangzhou, 2013, p. 1-6. DOI: 10.1109/WCSP.2013.6677217

[19] JIANG, T., YAO, W., SONG, Y., ET AL. Two novel nonlinear companding schemes with iterative receiver to reduce PAPR in multicarrier modulation systems. IEEE Transactions on Broadcasting, 2006, vol. 52, no. 2, p. 268-273. DOI: 10.1109/TBC.2006.872992

[20] PENG, S., SHEN, Y., YUAN, Z. PAPR reduction of multi-carrier systems with simple nonlinear companding transform. Electronic Letters, 2014, vol. 50, no. 6, p. 473-475. DOI: 10.1049/el.2013.4216

[21] COSTA, E., MIDRIO, M., PUPOLIN, S. Impact of amplifier nonlinearities on OFDM transmission systems performance. IEEE Communications Letters, 1999, vol. 3, no. 2, p. 37-39. DOI: $10.1109 / 4234.749355$

\section{About the Authors...}

SIMING PENG was born in Hubei, China Republic in 1990. He received his bachelor's degree from Wuhan University of Science and Technology in 2012. He is currently a M.Sc. candidate at the Department of Wireless Communications, College of Communications Engineering, PLA University of Science and Technology. His research interests include multicarrier communications and signal processing.

ZHIGANG YUAN was born in Hebei, China Republic in 1980. He received his Ph.D. degree in Communication and Information System from PLA University of Science and Technology in 2008. He is currently a lecture at the same university. His interests are signal processing and lattice multi-carrier communication theory.

JUN YOU was born in Jiangsu Province. He received the M.S. and Ph.D. degree from Institute of Communications Engineering, PLA University of Science and Technology, Nanjing, China, in 2001 and 2005, respectively. He is currently an associate professor in the College of Command Information System, PLAUST. His interest is fast signal processing.

YUEHONG SHEN was born in Hubei, China Republic in 1959. He received his Ph.D. degree in Communication Engineering from Nanjing University of Science and Technique in 1999. And now, he is a professor and doctor advisor of Wireless Department at the Institute of Communication Engineering, PLA University of Science and Technology, 
China. His interests include blind and source separation in communication systems.

WEI JIAN was born in Henan, China Republic in 1976. He received his Ph.D. degree in Communication and Information Engineering from PLA University of Science and Technology in 2006. His interests cover communication theory and high speed wireless communication systems. 